\title{
Assigning a Structural Motif using Spontaneous Molecular Dipole Orientation in Thin Films
}

\author{
M. Roman, A. Dunn ${ }^{(a)}$, S. Taj, Z. G. Keolopile ${ }^{(b)}$, A. Rosu-Finsen ${ }^{(c)}$, \\ M. Gutowski, and M. R. S. McCoustra, \\ Institute of Chemical Sciences, Heriot-Watt University, EDINBURGH, EH14 4AS, UK
}

\begin{abstract}
A. M. Cassidy and D. Field, Department of Physics and Astronomy, University of Aarhus, AARHUS, DK-8000, Denmark

(a) Current Address: Q2 Solutions, Alba Campus, Rosebank, LIVINGSTON, West Lothian, EH54 7EG, UK;

(b) Department of Physics, University of Botswana, Private Bag 0022, GABORONE, Botswana;

(c) Current Address: Department of Chemistry, University College London, LONDON, WC1H OAJ, UK.
\end{abstract}

\begin{abstract}
Spontaneous orientation of molecular dipoles has been observed to produce bulk electric fields, termed 'spontelectric' fields, in a broad variety of molecular solid thin films formed by condensation from the gas phase. Such spontelectric fields are found in cis-methyl formate (cis-MF) and the present work combines observation of these fields with high quality ab initio studies of cis-MF monomers and dimers. This enables a prediction of the structural motif within the unit cell of the crystalline phase of solid cis-MF, showing it to be a non-polar dimer. Dimer formation at deposition temperatures of $>90 \mathrm{~K}$ is cited to contribute to the observed collapse of the spontelectric field at these temperatures. This is the first time that such a structural prediction has been made, using observations of spontelectric behaviour as a key indicator.
\end{abstract}

\section{Introduction}

Powerful electric fields form in vapour deposited films of polar molecules and are the result of spontaneous orientation of the dipoles of the molecules involved [1 - 5]. The extent of dipole orientation, giving rise to this so-called 'spontelectric effect', has been described by an interplay between intermolecular forces, the electric field present in the material and the tendency to disorder introduced through thermal motion. This represents a classic orderdisorder system. Here, we introduce a new element into our understanding of the spontelectric phenomenon, suggesting that, as the temperature of deposition of films is raised, new structural motifs may arise which are themselves non-polar. Thus, the collapse 
of the spontelectric field at higher deposition temperatures may be viewed, in the case of cismethyl formate (cis-MF) at $\geq 90 \mathrm{~K}$, not only as a manifestation of competition between order and disorder, but also one in which new order additionally precludes the formation of the spontelectric state through the formation of non-polar structures.

We first briefly review the subject of spontelectrics. Observations of substantial potentials, on the surface of thin films of vapour deposited molecular solids, were first reported by Kutzner in 1972 and attributed to spontaneous dipole orientation of the constituent molecules [1]. These observations, however, remained largely ignored until such potentials, and corresponding dipole orientation in solids, were independently discovered by Field and co-workers in 2009, using surface potential measurements employing near-zero kinetic energy electrons [2]. This and later work established the presence of bulk electric fields, of strength exceeding $10^{8} \mathrm{~V} \mathrm{~m}^{-1}$, in thin films of nitrous oxide $\left(\mathrm{N}_{2} \mathrm{O}\right)$ grown at cryogenic temperatures of $38 \mathrm{~K}$ and above.

In subsequent studies, summarised in a number of reviews [3 - 5], Field and co-workers established the following salient observational properties of materials exhibiting such spontaneous dipole orientation:

(i) Surface potentials at the vacuum/film interface may be positive or negative.

(ii) The spontelectric field depends on the temperature at which the film is deposited.

(iii) Warming of a spontelectric film reveals a Curie temperature, at which the spontelectric effect decays abruptly. Subsequent cooling to below the Curie point has not been observed to reintroduce a surface potential.

(iv) The nature of the surface, upon which spontelectric films are deposited, has essentially no bearing on the values of surface potential.

(v) If the parent gas is diluted in a non-dipolar gas, a decrease is observed in the spontelectric field, to zero at sufficient dilution.

(vi) Spontelectric films may undergo a phase change whilst preserving their spontelectric character.

Field and co-workers were able to develop a simple parameterized order-disorder analysis explaining the resulting spontelectric fields, in films of materials deposited from vacuum, in terms of the temperature-dependent degree of dipole orientation in the solid material [3 - 5], verifying Kutzner's original supposition. Combining this analysis with experimental result for about a dozen small species of disparate structure, ranging from $\mathrm{CO}$ to methyl formate to 
toluene, the spontelectric effect has been shown to be both non-local and non-linear. The non-local character of spontelectrics is illustrated for example by data for toluene, which show some minimum thickness, for example of 90 monolayers (ML) deposited at $70 \mathrm{~K}$, to become spontelectric. This intriguing behaviour, which remains without explanation, indicates that, by some means, the material is aware of the presence of the 90 underlying layers, which collectively cause the character of thicker films to change [3 - 5]. The nonlinearity inherent in spontelectrics, see below, is best illustrated by studies of cis-methyl formate $[6,7]$, the subject of the present work.

Exploration of the spontelectric nature of $\mathrm{N}_{2} \mathrm{O}$ allowed identification of the impact of the vibrational Stark Effect on LO-TO splitting of molecular vibrations in thin solid films as an alternative means of identifying spontelectric materials [8] and demonstrated the potential of neutron scattering as a structural probe of the spontelectric phase [9]. With a simple infrared spectroscopic characterisation method available, the spontelectric nature of carbon monoxide (CO) thin films was demonstrated [10]. This turn furnished an explanation for the longstanding problem in the electronic spectroscopy of solid CO [11], and other species (e.g. ammonia [12]), of substantial band origin shifts with deposition temperature through the impact of the electronic Stark Effect on Wannier-Mott excitons within a spontelectric solid. The impact of the spontelectric effect on star formation and its potential for engineering electric field structures has also been explored [3 -5$]$. In addition, the impetus of the substantial body of work by Field and co-workers [ $3-5$ and references therein] has prompted others into investigating this novel, electrical phase of molecular materials [13, 14].

However, while we have made substantial strides in characterising molecular solids exhibiting this novel behaviour, we have as yet to identify more precisely the molecular motifs and balance of intermolecular interactions that necessarily drive this non-linear and non-local phenomenon. Evidence does, however, point to the observation that systems exhibiting strong hydrogen bond interactions may not behave spontelectrically where the directionality of the hydrogen bond dominates. Water nicely illustrates this, with observations from Field and co-workers that point to the absence of spontelectric behaviour in amorphous solid water at deposition temperature above $40 \mathrm{~K}$ [15] while $\mathrm{Bu}$ et al. [13] suggest that lower deposition temperatures, where the hydrogen bond connectivity is significantly reduced, result in a material which essentially behaves spontelectrically. The observations on butan-1-ol [14] would support this. Within the body of work by Field and coworkers, cis-methyl formate (cis-MF) and ethyl formate (EF) are representative of species which behave spontelectrically and reveal weak hydrogen bond interactions via the formate 
ester motif $[3-5,12,13]$. Cis-MF also exhibits unusual spontelectric behaviour in that the measured spontelectric field is first observed to decrease as deposition temperature is increased up $77 \mathrm{~K}$ in the normal manner but above $77 \mathrm{~K}$ is then observed to increase in a manner contrary to normal spontelectric behaviour before the spontelectric field disappears completely above $90 \mathrm{~K}$. This counter-intuitive, non-linear behaviour was predicted by the model of Field et al. and serves as a powerful indicator that this model captures some of the essential physics of the spontelectric effect. The mathematical form of the spontelectric model reproduces anomalous data for cis-methyl formate in which the degree of dipole orientation increases at deposition temperatures $>77 \mathrm{~K}$. However, only a qualitative physical explanation is presently available for this behaviour, which appears at first sight to contradict a simple order-disorder model [3].

A detailed infrared (IR) study of cis-methyl formate has also revealed this bi-modal behaviour with temperature below and above $77 \mathrm{~K}$ and is entirely consistent with the non-linear and non-local growth of spontelectric materials [7].

The current work complements the work reported in reference [7] and demonstrates, for the first time, the clear potential of spontelectric measurements in helping to reveal the structural motif inherent in a solid phase. While the IR spectroscopy of thin films of methyl formate is relatively well known $[16,17]$, the absence of any structural data has made interpretation of the spectra as a function of deposition temperature more complex. In this paper, we will demonstrate how knowledge of the spontelectric behaviour of MF, in particular the collapse of the spontelectric phenomenon at deposition temperatures $\geq 90 \mathrm{~K}$, can directly inform on the structure of the solid state.

For completeness, we should also note that dipole orientation has also been identified in organic light-emitting diode materials [18 and references therein]. Such films have electrical properties quite distinct from those of spontelectrics. Partial ordering, in these cases of large organics, appears to be based on local non-bonded interactions of the molecules with the substrate, rather than on the dipole-dipole interactions [19]. In this connection the spontelectric phenomenon is also quite distinct from the ferroelectric effect, as discussed at length in reference 3.

\section{Experimental and Computational Methodology}

Reflection-absorption IR (RAIR) spectra of MF in the spectral range 700 to $4000 \mathrm{~cm}^{-1}$, were obtained at Heriot-Watt University using essentially the same method as reported for $\mathrm{N}_{2} \mathrm{O}$ 
[2]. The experiments were conducted in an ultrahigh vacuum (UHV) chamber equipped with a line-of-sight quadrupole mass spectrometer (QMS, Hiden Analytical, HAL 3F/PIC) and a Fourier-transform infrared spectrometer (Varian 670-IR) configured for RAIR spectroscopy, at a grazing incidence of $75^{\circ}$ with respect to the normal to the substrate. After reflection from the sample, the IR beam was focused into a liquid nitrogen cooled $\mathrm{HgCdTe}$ detector. The base temperature of the system achieved using a closed-cycle helium refrigerator (APD Cryogenics, HC-2) was $18 \mathrm{~K}$, measured using a KP-type thermocouple. The substrate for MF film growth was oxygen-free, high conductivity copper, coated with a $300 \mathrm{~nm}$ layer of amorphous silica. The presence of amorphous silica allows the detection of TO modes [2] and spectra, taken at a resolution of $0.1 \mathrm{~cm}^{-1}$, hence show LO-TO splitting, key for the analysis involving the spontelectric effect. Spectral splitting is referred to throughout as LOTO splitting, whilst recognizing that the absolute value of the splitting arises through a combination of the intrinsically different vibrational frequencies associated with LO and TO modes and an independent contribution due to the vibrational Stark effect.

Liquid phase IR spectra were recorded using the attenuated total reflectance (ATR) technique using a Thermo Nicolet iS5 FTIR spectrometer fitted with an iD7 ATR accessory. The spectrum reported results from the co-addition of 16 spectral scans at a resolution of 1 $\mathrm{cm}^{-1}$. This gives us a set of IR spectra unperturbed by the effects of the solid state for comparison.

We recognize that the absolute energies of the individual molecules and that of the dimers (see below) will be affected by the presence of other surrounding species in the solid state. However, viewing this influence as a uniform perturbation which permeates the medium in a simple isotropic manner, we make the assumption here that the differences between the energies of the monomer and the dimers in the solid state may be represented by the differences for isolated species, as calculated here. On this basis, isolated MF was subject to investigation by electronic structure calculations at the coupled cluster level of theory [20] with single and double excitations (CCSD/aug-cc-pVTZ (ATZ) [21, 22]) in geometry optimizations and harmonic frequency calculations, and additional non-iterative triple excitations $(\operatorname{CCSD}(T))$ in single-point energy calculations. The SCF and MP2 energies were extrapolated to the basis set limit [23]. The dipole moments were calculated with the CCSD/ATZ densities. Finally, anharmonic corrections to vibrational frequencies were calculated at the MP2/ATZ level [24].

The MF monomer is equipped with a strong proton acceptor, but it lacks a strong proton donor. We expect the MF dimers to be only weakly bound, and therefore computational 
results might be contaminated with basis set superposition errors. Thus the geometries and harmonic frequencies of MF dimers were characterized on the counterpoise-corrected potential energy surface at the MP2/aug-cc-pVDZ (ADZ) [21, 22] level. We have considered 43 initial orientations of the monomers, including cis-cis, cis-trans, and trans-trans complexes. As proton donors we have considered both the $\mathrm{CH}_{3}$ and $\mathrm{CH}$ hydrogens, and as proton acceptors both the carbonyl and ester oxygens. The geometry optimizations initiated from these 43 initial structures converged to 16 structures shown in the electronic supplementary information (ESI), Figure S1. The cis-cis and trans-trans complexes were the most and least stable, respectively. Thus the cis-cis complexes were studied more thoroughly. The total stabilization energy of the dimer was calculated as a sum of two onebody terms (repulsive terms resulting from deformations of the monomers in the dimer) and an attractive two-body term (the interaction energy between two deformed monomers), see reference [25].

\section{Results and Discussion}

\section{Methyl Formate Rotational Isomerism in the Gas Phase}

MF is subject to rotational isomerism around both the methyl - oxygen and the formyl oxygen bonds, i.e. the dihedral angles $\mathrm{H}-\mathrm{CH}_{2}-\mathrm{O}-\mathrm{C}$ and $\mathrm{CH}_{3}-\mathrm{O}-\mathrm{C}=\mathrm{O}$. Internal rotation about the latter is the most significant with electronic structure results shown in Figure 1 indicating that cis-MF is $19.7 \mathrm{~kJ} \mathrm{~mol}^{-1}$ more stable than trans-MF with the separating barrier of $52.7 \mathrm{~kJ}$ $\mathrm{mol}^{-1}$. The polarity increases from cis-MF to trans-MF, 1.84 D and 4.26 D, respectively. The geometries of these three stationary points are tabulated in the ESI, Table S1.

The MP2 and CCSD harmonic vibrational constants and MP2 anharmonic corrections for the 0-1 transition for cis-MF, trans-MF, and the transition state are tabulated in the ESI, Tables S2-S4, whereas here we focus on the $\mathrm{C}=\mathrm{O}$ stretching mode only, which has the highest intensity in the cis- and trans-MF. The calculated CCSD harmonic vibrational constant is 1827 and $1869 \mathrm{~cm}^{-1}$ and the 0-1 anharmonic transition is predicted at 1796 and $1841 \mathrm{~cm}^{-1}$ for the gas phase cis- and trans-MF, respectively, see Table 1. The results for cis-MF are in good agreement with the experimental gas phase spectrum [26]. From here on, we consider only this readily identifiable $\mathrm{C}=\mathrm{O}$ stretching mode.

Simple Boltzmann analysis then suggests the trans-form comprises significantly less than 1 part in $10^{8}$ of the total in the gas phase up to $120 \mathrm{~K}$. In view of a high barrier separating cis from trans, and weak intermolecular interactions between MF molecules reflected by the 
melting point of MF of $173 \mathrm{~K}$, we assume that the spontelectric measurements and IR spectroscopy exclusively probe cis-MF in thin films.

\section{Dimers of Methyl Formate}

Sixteen low-energy structures of the MF dimer (D1-D16) are illustrated in the ESI, Figure S1. In view of the weakness of the intermolecular interactions, the relative energies and stabilization energies are given in $\mathrm{meV}\left(1 \mathrm{meV}=0.0965 \mathrm{~kJ} \mathrm{~mol}^{-1}\right)$. The results confirm that the carbonyl oxygen is a stronger proton acceptor than the ester oxygen. There are only minor stability preferences for the dimers, with proton donors resulting from $\mathrm{CH}_{3}$ rather than from $\mathrm{CH}$. The eight most stable dimers are assembled from two cis monomers. The lowest energy dimer involving at least one trans monomer, D9, is less stable than D1 by $182 \mathrm{meV}$ $\left(=17.6 \mathrm{~kJ} \mathrm{~mol}^{-1}\right)$.

Hereafter, we focus on the three most stable dimers of MF, D1-D3, illustrated in Figure 2. Other dimeric structures are less stable by at least $30 \mathrm{meV}$ at the MP2/ADZ level. Both D1 and D3 are symmetric $\left(\mathrm{C}_{2 h}\right)$ and the small difference in stability $(14 \mathrm{meV})$ results from a difference in the nature of the proton donors: $\mathrm{CH}_{3}$ and $\mathrm{CH}$, respectively. Not surprisingly, D2 has one $\mathrm{CH}_{3}$ and one $\mathrm{CH}$ proton donor and its stability is in between D1 and D3, still $10 \mathrm{meV}$ less stable than D1. D1 and D3 have overall zero dipole moments by symmetry, while the MP2 dipole moment of D2 is $0.75 \mathrm{D}$ at the MP2/ADZ level. The total stabilization energies in D1-D3 are dominated by attractive two-body components (from -199 to -183 meV), with repulsive one-body deformation components for the cis monomers not exceeding $5 \mathrm{meV}$, see Table 2. The zero-point vibrational corrections are destabilizing for all three dimers (between 18 and $27 \mathrm{meV}$ ), in agreement with our previous discussion [25]. Due to the weak hydrogen bonding, the $\mathrm{C}=\mathrm{O}$ stretching modes are red-shifted in comparison with the isolated monomer; see Table S5 in the ESI. The shift is more substantial for the symmetric than for the asymmetric $\mathrm{C}=\mathrm{O}$ stretching mode. The asymmetric and symmetric modes become split by 10,14 , and $13 \mathrm{~cm}^{-1}$ in D1, D2, D3, respectively.

\section{Spontelectric Measurements on cis-MF Thin Films}

Figure 3(a) shows the variation of spontelectric field in cis-MF thin films as a function of deposition temperature taken from references [3 - 7]. The highly anomalous behaviour expressed in this system of the degree of dipole orientation, and hence the spontelectric field, first decreasing with increasing deposition temperature then increasing as the temperature increases above $77 \mathrm{~K}$ is clearly observed. Explanation of this phenomenon with 
the context of non-linear and non-local growth of the spontelectric field in cis-MF films has been given [7]. In this work, we focus on the observation that above $90 \mathrm{~K}$, no spontelectric field can be measured. Within the framework presented by Field and co-workers to explain the origin of spontelectric behaviour [3 - 5], this can be interpreted as meaning that either the molecular dipoles are completely randomly oriented in an amorphous solid phase, or alternatively, that $90 \mathrm{~K}$ represents a phase transition point and the crystalline solid phase prepared by deposition above $90 \mathrm{~K}$ presents a lattice motif in which the molecular dipoles are in a centrosymmetric structure.

\section{Infrared Spectroscopy of cis-MF Thin Films}

Table 3 summarises our assignment of the IR spectrum of cis-MF in comparison to the reported work in references [14] and [15]. A sharpening of the $\mathrm{C}=\mathrm{O}$ line profile at around $1720 \mathrm{~cm}^{-1}$, and of those of the other vibrational modes, is observed between 90 and $100 \mathrm{~K}$. This is consistent with previous work from the literature $[25,26]$ and suggests that at the higher deposition temperatures, the resulting film is crystalline in nature.

Figure 4 presents experimental data recorded for films deposited at 70,90 and $108 \mathrm{~K}$ to illustrate this behaviour and our analysis of LO-TO splitting of the $\mathrm{C}=\mathrm{O}$ stretching mode, where the LO modes is observed to lie between 1733.8 and $1736.8 \mathrm{~cm}^{-1}$ while the TO modes lie between 1710.8 and $1713.2 \mathrm{~cm}^{-1}$. Figure $3(\mathrm{~b})$ summarises the corresponding LO-

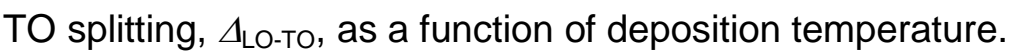

In the amorphous phase $(<90 \mathrm{~K})$, a single LO-TO pair is observed. In the crystalline phase (> $90 \mathrm{~K}$ ), additional features consistent with a second LO-TO pair are observed. Comparing Figure 3(a) and (b), we can see immediately that (1) below $90 \mathrm{~K}$, are changes in the LO-TO splitting that can be successfully explained by the presence of a spontelectric field and the vibrational Stark Effect [7]. Above $90 \mathrm{~K}$, however, the absence of a spontelectric field means that the vibrational Stark Effect is switched off and the LO-TO splitting remains constant. Indeed, both LO-TO pairs observed in the crystalline phase at these temperatures show constant splitting as the temperature is increased beyond the crystallisation point.

Figure 5 compares the crystalline phase $\mathrm{C}=\mathrm{O}$ stretching features from previous reports on this system $[16,17]$ with that of the current study. The liquid phase spectrum lacks any fine structure and illustrates the situation of truly randomly orientated dipoles. The RAIR spectrum from Brown and co-workers [17] (blue) reports measurements made on a graphite substrate and therefore subject to the metal surface selection rule. Consequently only the 
LO mode of the LO-TO split pair is observed. The RAIR spectra recorded in this work (black) and by transmission spectra of Palumbo and co-workers [16] (red) are remarkably similar in showing both components of the LO-TO split of $\mathrm{C}=\mathrm{O}$ stretching mode. This illustrates the fortuitous advantage conferred in relation to the present study of the $200 \mathrm{~nm}$ silica nanoparticulate film present on the copper substrate surface in allowing us to circumvent the metal surface selection rule.

So how do we explain the additional LO-TO pair in the crystalline phase? The absence of any spontelectric field in the high temperature regime could point to complete randomisation of the dipole orientation but that would be inconsistent with a crystalline solid. Could we be observing the presence of both cis and trans rotational isomers in the crystalline phase? No! Comparison of the computed anharmonic frequencies in Table 1 with the observed frequencies in the crystalline phase clearly reveals that the shift between cis and trans $\mathrm{C}=\mathrm{O}$ stretching modes, at around $45 \mathrm{~cm}^{-1}$, is substantially more than we observe in the experimental spectra.

Alternatively, we can interpret the absence of the spontelectric field to suggest that the molecular dipoles may be oriented in a non-dipolar, centrosymmetric motif in the crystalline phase. A ring dimer motif, as suggested in Figure 2, could provide the explanation. The two LO-TO split modes therefore representing symmetric and anti-symmetric coupled $\mathrm{C}=\mathrm{O}$ vibrations within the ring motif. The computational investigation of ring dimer structures of MF presented above supports this latter proposal. Indeed, the computed splitting in the symmetric and anti-symmetric coupled $\mathrm{C}=\mathrm{O}$ vibrations in the ring structures $\mathrm{D} 1, \mathrm{D} 2$, and D3 are 10,14 , and $13 \mathrm{~cm}^{-1}$, respectively. While, the experimental IR spectra in Figure $5(\mathrm{c})$ show a very broad band $C=0$ stretch made of four peaks at $\approx 1719, \approx 1710, \approx 1703$ and $\approx 1697$ $\mathrm{cm}^{-1}$. These peaks had to be coupled into two LO-TO pairs to represent the split symmetric and split asymmetric modes. An LO-TO pair of 1719 and $1703 \mathrm{~cm}^{-1}$ would give a midpoint at $1711 \mathrm{~cm}^{-1}$ and a pair of 1710 and $1697 \mathrm{~cm}^{-1}$ would give one at $1704 \mathrm{~cm}^{-1}$. These are consistent with the positions from Palumbo's work at $110 \mathrm{~K}\left(\approx 1713\right.$ and $\left.\approx 1707 \mathrm{~cm}^{-1}\right)$ [25], and the positions for the LO peaks from Brown's data [17]. Hence, an experimental splitting of $7 \mathrm{~cm}^{-1}$ is consistent with that computed for structure D1.

\section{Conclusions}

In conclusion, a combination of measurements of spontaneous dipole orientation, IR spectroscopy and computational chemistry supports the proposal that the basis motif of the lattice in crystalline cis-methyl formate is a ring dimer structure. This work would represent 
the first time such a combination of observations have been employed in structural identification in the solid state and shows how the spontelectric effect may be used to point the way to the identification of new structures in solid material.

\section{Acknowledgements}

MM wishes to acknowledge the supported of the U.K. Science and Technology Facilities Council (STFC, ST/M001075/1), the U.K. Engineering and Physical Sciences Research Council (EPSRC, GR/T27044/02) and the European Community FP7-ITN and H2020-ITN Marie Curie Programmes (LASSIE Project, Grant Agreement 238258; and EuroPAH Project, Grant Agreement 722346). We also acknowledge use of resources of the National Energy Research Scientific Computing Centre (NERSC), which is supported by the Office of Science of the U.S. Department of Energy under Contract No. DE-AC02-05CH11231.

\section{References}

[1] K. Kutzner, Spontaneous Polarization of condensing Carbon Monoxide and other Gases with an Electric Dipole Moment. Thin Solid Films, 1972, 14, 49-61. (DOI: 10.1016/00406090(72)90369-0)

[2] R. Balog, P. Cicman, N. C. Jones, and D. Field, Spontaneous Dipole Alignment in Films of $\mathrm{N}_{2}$ O. Phys. Rev. Lett., 2009, 102, 073003.(DOI: 10.1103/PhysRevLett.102.073003)

[3] D. Field, O. Plekan, A. N. Cassidy, R. Balog, N. C. Jones, and J. Dunger, Spontaneous Electric Fields in Solid Films: Spontelectrics. Int. Rev. Phys. Chem., 2013, 32, 345-392. (DOI: 10.1080/0144235X.2013.767109)

[4] O. Plekan, A. Rosu-Finsen, A. M. Cassidy, J. Lasne, M. R. S. McCoustra, and D. Field, A Review of Recent Progress in Understanding the Spontelectric State of Matter. Eur. Phys. J. $D, 2017,71,162-180$. (DOI: 10.1140/epjd/e2017-80048-3)

[5] D. Field, Spontelectric Solids. Phil. Trans. Roy. Soc. A, submitted.

[6] O. Plekan, A. M. Cassidy, R. Balog, N. C. Jones, and D. Field, Spontaneous Electric Fields in Films of cis-Methyl Formate. Phys. Chem. Chem. Phys., 2012, 14, 9972-9976. (DOI: $10.1039 / c 2 c p 41229 b)$

[7] M. Roman, S. Taj, M. Gutowski, M .R. S. McCoustra, A. C. Dunn, Z. G. Keolopile, A. Rosu-Finsen, A. M. Cassidy, and D. Field, Non-linear and Non-local Behaviour in Spontaneously Electrical Solids. Phys. Chem. Chem. Phys., 2018, 20, 5112-5116. (DOI: 10.1039/c7cp08489g) 
[8] J. Lasne, A. Rosu-Finsen, A. M. Cassidy, M. R. S. McCoustra, and D. Field, Spontaneously Electrical Solids in a New Light. Phys. Chem. Chem. Phys., 2015, 17, 2097120980. (DOI: 10.1039/C5CP03174E)

[9] A. M. Cassidy, M. R. V. Jørgensen, A. Rosu-Finsen, J. Lasne, J. H. Jørgensen, A. Glavic, V. Lauter, B. B. Iversen, M. R. S. McCoustra, and D. Field, Dipole-Oriented Molecular Solids Can Undergo a Phase Change and Still Maintain Electrical Polarization. J. Phys. Chem. C., 2016, 120, 24130-24136. (DOI: 10.1021/acs.jpcc.6b07296)

[10] J. Lasne, A. Rosu-Finsen, A. M. Cassidy, M. R. S. McCoustra, and D. Field, Spontaneous Electric Fields in Solid CO. Phys. Chem. Chem. Phys., 2015, 17, 3017730187. (DOI: 10.1039/C5CP04536C)

[11] Y. -J. Chen, G. Muñoz Caro, S. Aparicio, A. Jiménez-Escobar, J. Lasne, A. RosuFinsen, M. R. S. McCoustra, A. M. Cassidy, and D. Field, Wannier-Mott Excitons in Nanoscale Molecular Ices. Phys. Rev. Lett., 2017, 119, 157703-157707. (DOI: 10.1103/PhysRevLett.119.157703)

[12], A. Dawes, R. J. Mukerji, M. P. Davies, P. D. Holtom, S. M. Webb, B. Sivaraman, S. V. Hoffmann, D. A. Shaw, and N. J. Mason, Morphological Study into the Temperature Dependence of Solid Ammonia under Astrochemical Conditions using Vacuum Ultraviolet and Fourier-transform Infrared Spectroscopy. J. Chem. Phys., 2007, 126, 244711. (DOI: 10.1063/1.2743426)

[13] C. Bu, J. Shi, U. Raut, E. H. Mitchell, and R. A. Baragiola, Effect of Microstructure on Spontaneous Polarization in Amorphous Solid Water Films. J. Chem. Phys., 2015, 142, 134702-134706. (DOI: 10.1063/1.4916322)

[14] I. K. Gavra, A. N. Pilidi, and A. A. Tsekouras, Spontaneous Polarization of Vapourdeposited 1-Butanol Films and its Dependence on Temperature. J. Chem. Phys., 2017, 146, 104701-14708. (DOI: 10.1063/1.4978239)

[15] R. Balog, P. Cicman, D. Field, L. Feketeová, K. Hoydalsvik, N. C. Jones, T. A. Field, and J. -P. Ziesel, Transmission and Trapping of Cold Electrons in Water Ice, J. Phys. Chem. A, 2011, 115, 6820-6824. (DOI: 10.1021/jp110475q)

[16] P. Modica and M. E. Palumbo, Formation of Methyl Formate after Cosmic Ion Irradiation of Icy Grain Mantles. Astron. Astrophys., 2010, 519, A22. (DOI: 10.1051/00046361/201014101)

[17] D. J. Burke, F. Puletti, P. M. Woods, S. Viti, B. Slater and W. A. Brown, Adsorption and Thermal Processing of Glycolaldehyde, Methyl Formate, and Acetic Acid on Graphite at 20 K. J. Phys. Chem. A, 2015, 119, 6837-6849. (DOI: 10.1021/acs.jpca.5b04010).

[18] K. Osada, K. Goushi, H. Kaji, C. Adachi, H. Ishii, and Y. Noguchi 2018, Observations of Spontaneous Orientation Polarization in Evaporated Films of Organic Light-emitting Diode Materials. Organic Electronics, 2018, 58, 313-317. (DOI: 10.1016/j.orgel.2018.04.026) 
[19] P. Friederich, V. Rodin, F. von Wrochem, and W. Wenzel. Built-In Potentials Induced by Molecular Order in Amorphous Organic Thin Films. ACS Appl. Mater. Interfaces, 2018, 10, 1881-1887. (DOI: 10.1021/acsami.7b11762)

[20] R. J. Bartlett and M. Musiał, Coupled-cluster Theory in Quantum Chemistry. Rev. Mod. Phys., 2007, 79, 291-352. (DOI: 10.1103/RevModPhys.79.291)

[21] T. H. Dunning, Gaussian Basis Sets for use in Correlated Molecular Calculations. I. The Atoms Boron through Neon and Hydrogen. J. Chem. Phys., 1989, 90, 1007-1023. (DOI: $10.1063 / 1.456153)$

[22] R. A. Kendall, T. H. Dunning, and R. J. Harrison, Electron Affinities of the First-row Atoms Revisited. Systematic Basis Sets and Wave Functions. J. Chem. Phys., 1992, 96, 6796-6806. (DOI: 10.1063/1.462569)

[23] R. A. Bachorz, W. Klopper, and M. Gutowski, Coupled-cluster and Explicitly Correlated Perturbation-theory Calculations of the Uracil Anion. J. Chem. Phys., 2007, 126, 085101. (DOI: 10.1063/1.2436890)

[24] V. Barone, V., Anharmonic Vibrational Properties by a Fully Automated Second-order Perturbative Approach. J. Chem. Phys., 2005, 122, 014108. (DOI: 10.1063/1.1824881)

[25] Z.G. Keolopile, M.R. Ryder, and M. Gutowski, Intermolecular Interactions between Molecules in Various Conformational States: The Dimer of Oxalic Acid. J. Phys. Chem. A, 2014, 118, 7385-7391. (DOI: 10.1021/jp4125638)

[26] https://webbook.nist.gov/cgi/cbook.cgi?Name=methyl+formate\&Units=SI\&clR=on (Last Accessed 03 August 2018).

[27] R. F. Curl, Microwave Spectrum, Barrier to Internal Rotation, and Structure of Methyl Formate. J. Chem. Phys., 1959, 30, 1529-1536. (DOI: 10.1063/1.1730232) 


\section{FIGURES}

Figure 1: Electronic energy profile for methyl formate (MF) along the dihedral $\mathrm{CH}_{3}-\mathrm{O}-\mathrm{C}=\mathrm{O}$ angle. Three stationary points are illustrated: cis-MF, transition state TS, and trans-MF, with the energies corrected for zero-point vibrations. The dipole moment increases from cis through TS to trans. The experimental dipole moment of cis-MF is $1.77 \mathrm{D}$ [27].

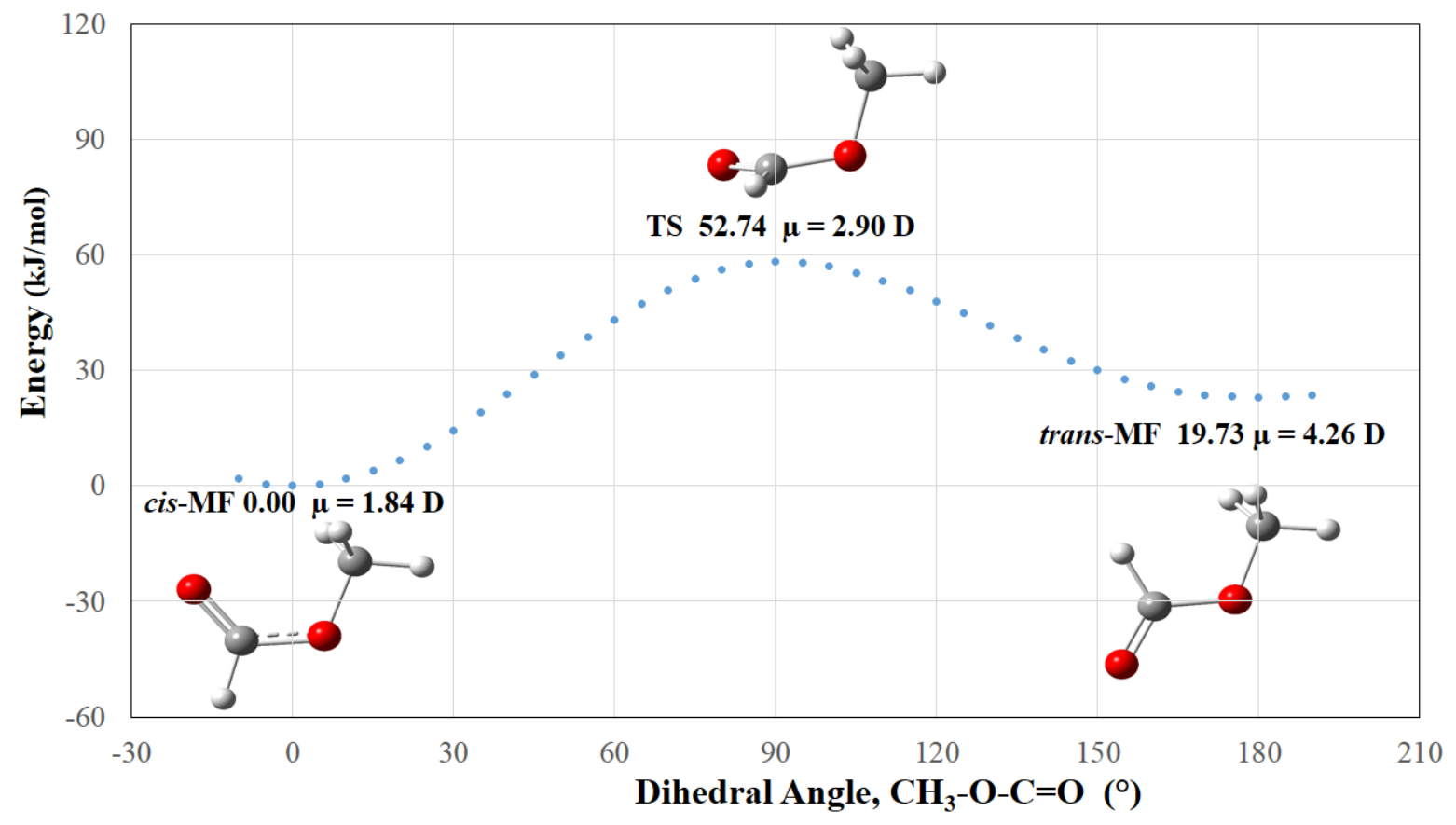


Figure 2: The three most stable dimers of MF. The relative energies are given in meV.

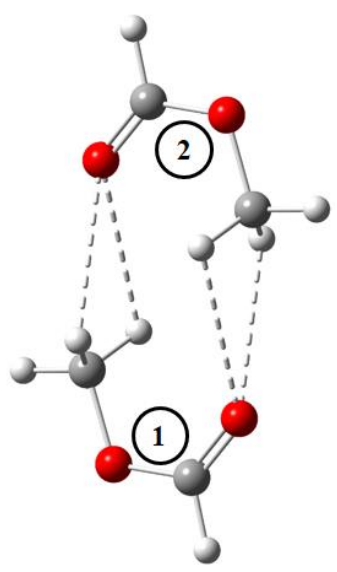

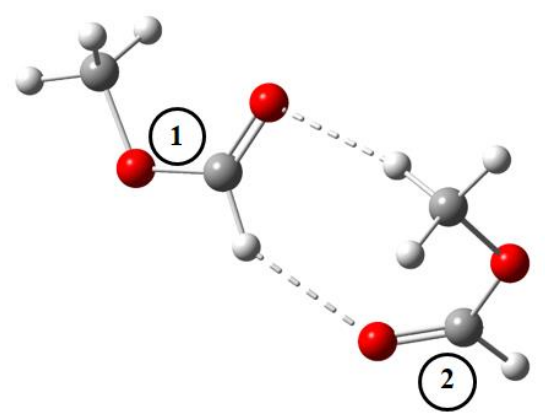

D2, $C_{1}, \mathbf{1 0 . 4 0}$

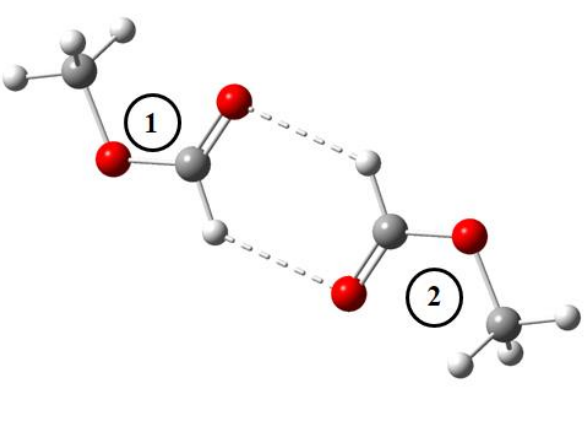

D3, $C_{2 h}, 13.66$ 
Figure 3: (a) The variation of the degree of dipole alignment, $\left\langle\mu_{z}>/ \mu\right.$, as a function of deposition temperature for the cis-MF film resulting from a $2000 \mathrm{~L}\left(1 \mathrm{~L} \equiv 10^{-6} \mathrm{mbar} \mathrm{s}\right)$ exposure to MF vapour at room temperature; (b) variation of LO-TO splitting, $\Delta_{\mathrm{LO}-\mathrm{TO}}$, as a function of deposition temperature for an equivalent film of cis-MF where the black squares present the splitting for a single $\mathrm{C}=\mathrm{O}$ mode as observed in the amorphous phase of cis-MF and the red circles/blue triangles represent the pair of $\mathrm{C}=\mathrm{O}$ stretching modes observed in the crystalline phase.

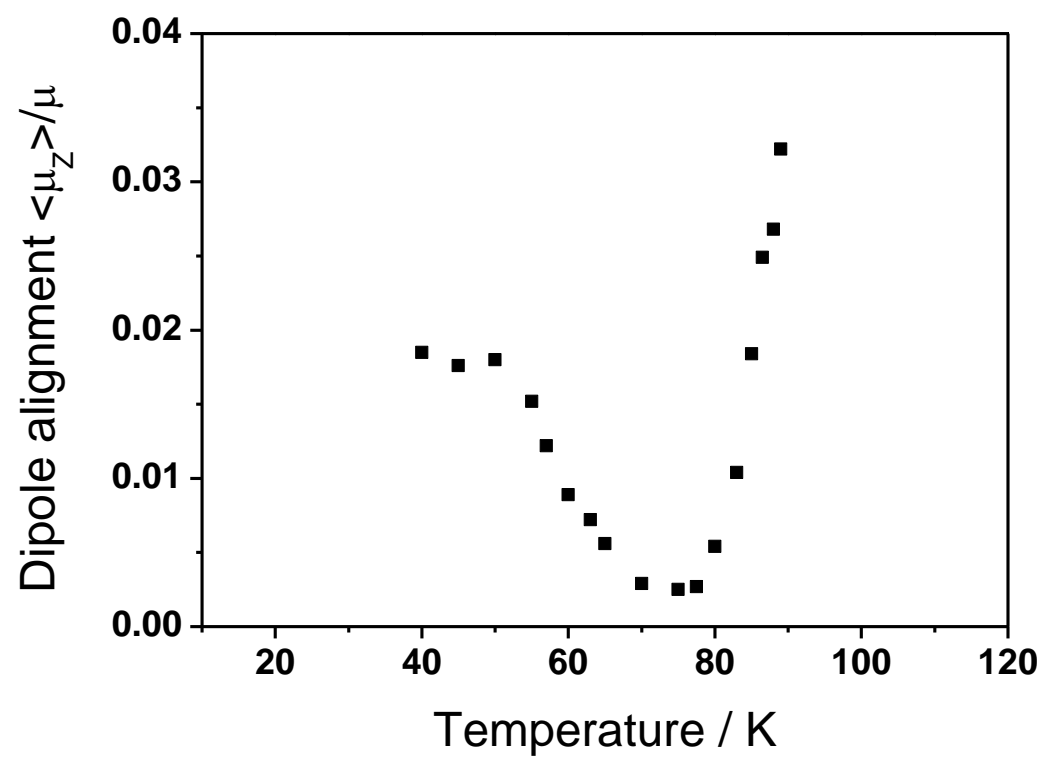

(a)

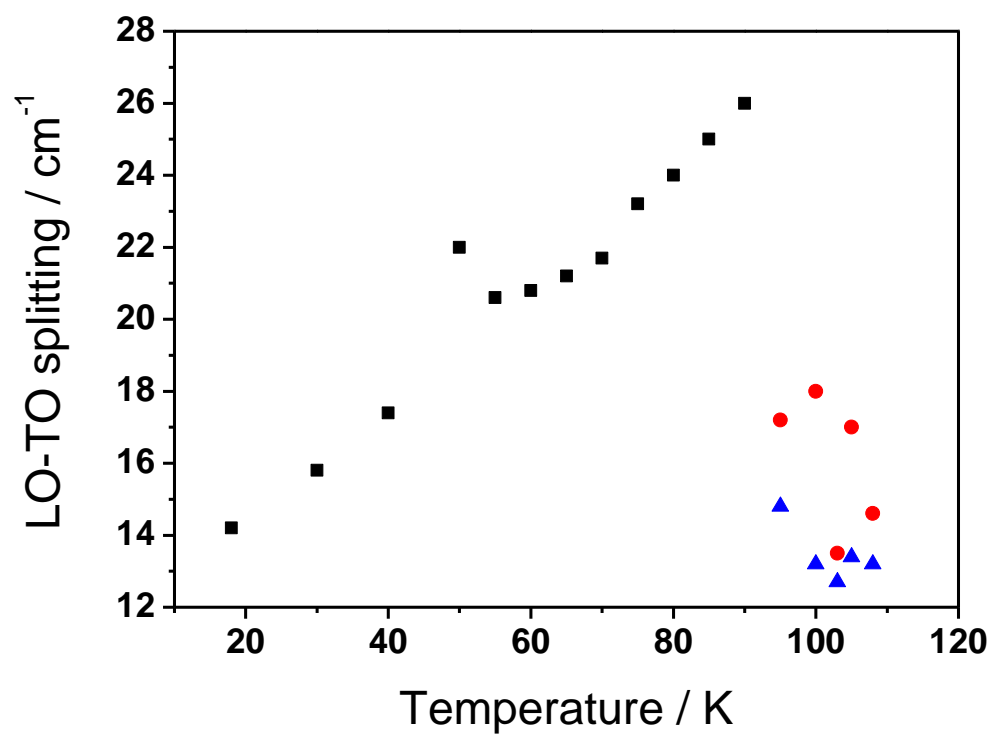


Figure 4: RAIR spectra of solid cis-MF deposited on an amorphous silica film on a copper surface (black lines) for films of few 10's of ML. The sharp spectral features, useful as wavelength markers, are due to residual water vapour in the purge gas of our external optics. Spectrum (a) was recorded at $70 \mathrm{~K}$ deposition temperature, spectrum (b) was obtained at $90 \mathrm{~K}$ and spectrum (c) at $108 \mathrm{~K}$. Spectra are the result of co-adding 512 scans at a resolution of $0.1 \mathrm{~cm}^{-1}$ in typically 60 minutes. The blue lines are Gaussian component fits to the LO and TO modes. As can be seen, the spectrum obtained at $108 \mathrm{~K}$ requires fitting with an additional two components compared to those at 70 and $90 \mathrm{~K}$. The corresponding LO-TO splitting as a function of deposition temperature are shown in Figure 3(b). The red dashed line shows the sum of the fits, the greater discrepancy at $90 \mathrm{~K}$ compared with $70 \mathrm{~K}$ indicating increasingly disordered molecular structure at higher deposition temperature.

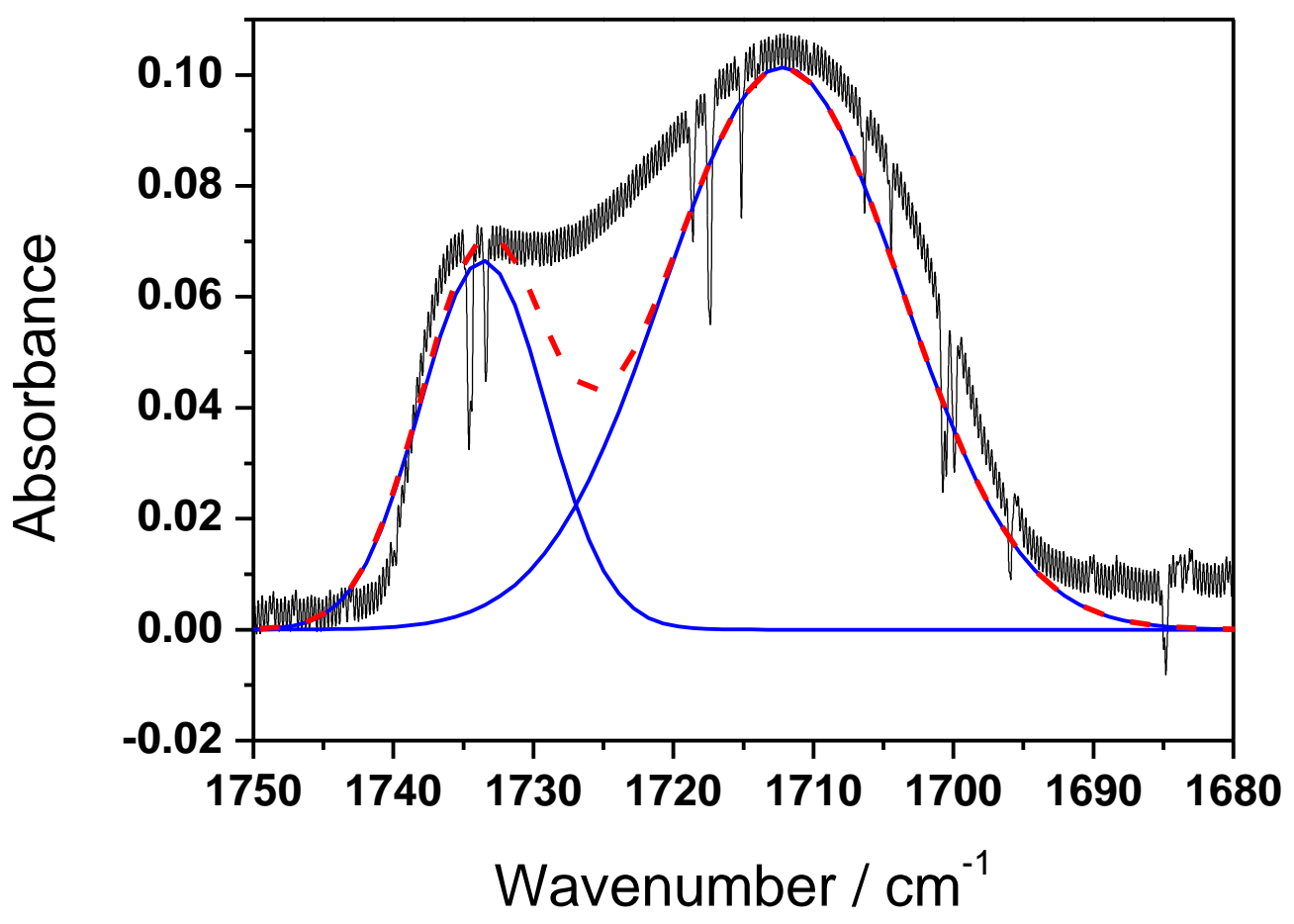



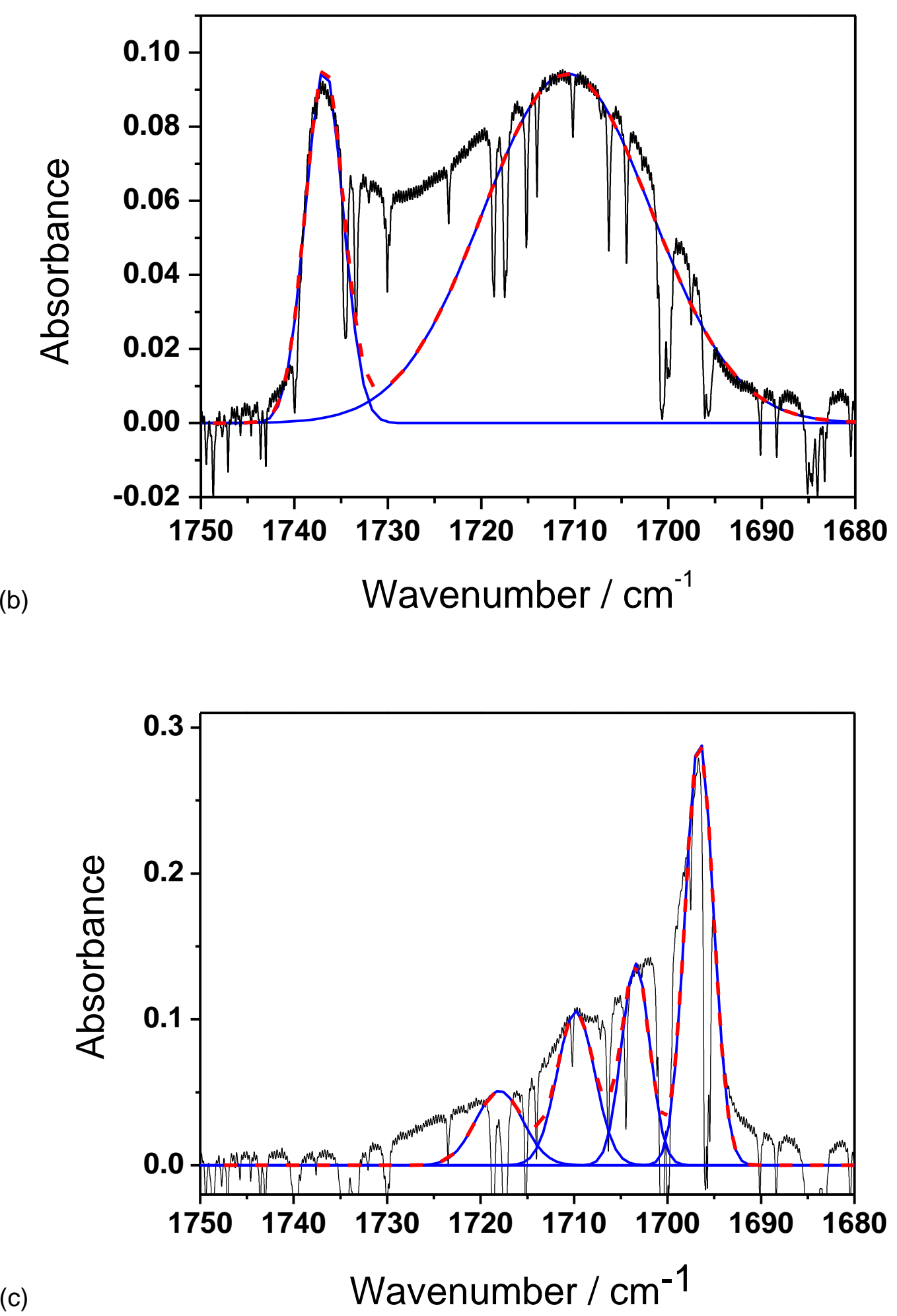
Figure 5: The $\mathrm{C}=\mathrm{O}$ stretching band of liquid (purple) and crystalline cis-MF from the present RAIRS study (black) and previously reported transmission studies by Palumbo and coworkers [14] (red) and RAIRS studies from Brown and co-workers [15] (blue). The narrow features in the black spectrum are a result of variation of the gas phase water content in dry air used to purge the external optics attached to the spectrometer and UHV system. Figure 4 (c) illustrates how even with the strong, but narrow, water features the black spectrum can be fitted to four components corresponding to the in-phase and out-of-phase LO-TO split $\mathrm{C}=\mathrm{O}$ stretching mode.

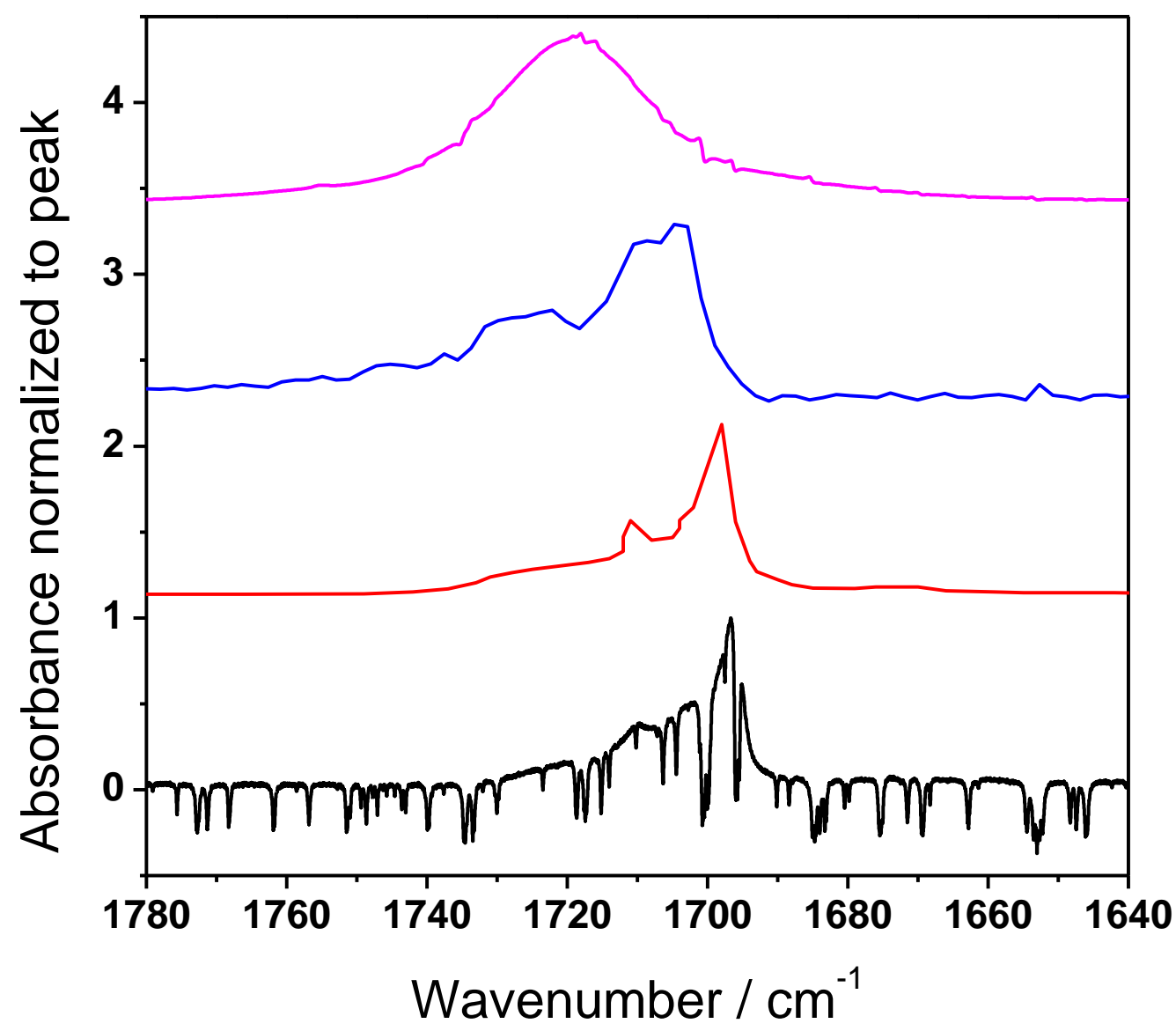




\section{TABLES}

Table 1: Harmonic $(\mathrm{H}) \mathrm{C}=\mathrm{O}$ vibrational constant, anharmonic $(\mathrm{AH})$ correction to the $0-1$ transition, and anharmonic 0-1 transition $\left(\mathrm{cm}^{-1}\right)$ in cis- and trans-MF. $\delta \mathrm{MP} 2(\mathrm{AH})$ stands for anharmonic correction obtained at the MP2 level. All results obtained with the ATZ basis set.

\begin{tabular}{|c|c|c|c|c|c|}
\hline Structure & \multicolumn{5}{|c|}{$\mathrm{C}=\mathrm{O}$ Vibrational mode } \\
\hline & $\operatorname{CCSD}(\mathrm{H})$ & MP2(H) & $\mathrm{MP2}(\mathrm{AH})$ & $\delta \mathrm{MP} 2(\mathrm{AH})$ & $\begin{array}{l}\operatorname{CCSD}(\mathrm{H})+ \\
\delta \mathrm{MP} 2(\mathrm{AH})\end{array}$ \\
\hline cis & 1827.0 & 1769.5 & 1738.5 & -31.0 & 1796.0 \\
\hline trans & 1869.1 & 1807.1 & 1778.5 & -28.5 & 1840.6 \\
\hline
\end{tabular}


Table 2: Total stabilisation energy in the D1-D3 dimers represented as a sum of one-body components (monomer deformation terms), two-body interaction energy between deformed monomers, and a zero-point vibration energy contribution (all terms in meV).

\begin{tabular}{|c|c|c|c|c|c|c|}
\hline Dimer & Monomer & $E_{c i s-M F}^{1 b-d e f}$ & $E_{1 \leftrightarrow 2}^{2 b}$ & $E_{1 \leftrightarrow 2}^{S t a b}$ & $\Delta E_{0}^{v i b}$ & $E_{1 \leftrightarrow 2}^{\text {Stab }}+E_{0}^{\text {vib }}$ \\
\hline \multirow{2}{*}{ D1 } & 1@D1 & 2.30 & \multirow{2}{*}{-199.03} & \multirow{2}{*}{-194.43} & \multirow{2}{*}{25.47} & \multirow{2}{*}{-168.96} \\
\hline & 2@D1 & 2.30 & & & & \\
\hline \multirow{2}{*}{ D2 } & 1@D2 & 4.64 & \multirow{2}{*}{-194.47} & \multirow{2}{*}{-185.95} & \multirow{2}{*}{27.40} & \multirow{2}{*}{-158.56} \\
\hline & 2@D2 & 3.88 & & & & \\
\hline \multirow{2}{*}{ D3 } & 1@D3 & 4.65 & \multirow{2}{*}{-182.93} & \multirow{2}{*}{-173.63} & \multirow{2}{*}{18.34} & \multirow{2}{*}{-155.30} \\
\hline & 2@D3 & 4.65 & & & & \\
\hline
\end{tabular}


Table 3: Comparison of the vibrational assignment of the IR spectrum of solid cis-MF from this work and from that of Palumbo and co-workers [14] and Brown and co-workers [15]. The amorphous cis-MF films were deposited at $16 \mathrm{~K}$ [14], $26 \mathrm{~K} \mathrm{[15]} \mathrm{and} \mathrm{at} 18 \mathrm{~K}$ in this work. Spectra of crystalline cis-MF were deposited at $110 \mathrm{~K}$ [14], $105 \mathrm{~K}$ [15] and $108 \mathrm{~K}$ in this work.

\begin{tabular}{|c|c|c|c|c|c|c|}
\hline & \multicolumn{3}{|c|}{ Amorphous cis-MF peak positions } & \multicolumn{3}{c|}{ Crystalline cis-MF peak positions } \\
\hline Vibration & {$[\mathbf{1 4}]$} & {$[\mathbf{1 5}]$} & This Work & {$[\mathbf{1 4}]$} & {$[\mathbf{1 5}]$} & This Work \\
\hline $\mathrm{CH}_{3}$ stretch & 3038,3010 & 3015 & 3011 & & 3013,3019 & 3012 \\
\hline $\mathrm{CH}$ stretch & 2960 & 2964 & 2959 & & 2963,2978 & 2961,1976 \\
\hline $\mathrm{C}=\mathrm{O}$ stretch & 1720 & 1736 & 1734,1720 & 1713,1707 & 1724,1706 & $\begin{array}{c}1717,1706, \\
1710,1697\end{array}$ \\
\hline $\begin{array}{c}\mathrm{CH}_{3} \\
\text { deformation }\end{array}$ & 1450,1453 & 1450,1460 & 1453 & 1450,1453 & $\begin{array}{c}1440,1451, \\
1459,1472\end{array}$ & $\begin{array}{c}1440,1454, \\
1460\end{array}$ \\
\hline $\begin{array}{c}\mathrm{CH} \text { bend } \\
\mathrm{C}-\mathrm{O} \text { stretch }\end{array}$ & 1383 & 1388 & 1389 & & 1394 & 1392 \\
\hline $\begin{array}{c}\mathrm{CH}_{3} \text { rock } \\
\begin{array}{c}\mathrm{O}-\mathrm{CH} \\
\text { stretch }\end{array}\end{array}$ & 1164 & 1173 & 1171 & 1164 & 1164,1177 & 1166,1173 \\
\hline $\begin{array}{c}\mathrm{OCO} \\
\text { deformation }\end{array}$ & 710 & 910 & 913 & 910 & 901,906 & 899,905 \\
\hline
\end{tabular}

